\title{
Comparative Studies on Solubility and Dissolution Enhancement of Different Itraconazole Salts and Their Complexes
}

\author{
Hemalatha Bagavatula ${ }^{1}$, Srinivas Lankalapalli ${ }^{1, *}$, VS Vinai Kumar Tenneti ${ }^{1}$, \\ Naga Malleswara Rao Beeraka ${ }^{2}$, Bhanu Teja Bulusu ${ }^{2}$ \\ ${ }^{1}$ GITAM Institute of Pharmacy, GITAM University, Rushikonda, Visakhapatnam, Andhra Pradesh, India \\ ${ }^{2}$ There Dose Pharma Pvt.Ltd, ALEAP Industrial Estate, Pragathi Nagar, Kukatpally, Hyderabad, Andhra Pradesh, India
}

Copyright (C) 2014 Horizon Research Publishing All rights reserved.

\begin{abstract}
Itraconazole is a potent triazole antifungal drug which has low solubility at physiological $\mathrm{pH}$ conditions. Itraconazole is weakly basic ( $\mathrm{pKa}=3.7$ ) and highly hydrophobic drug. It is categorized as a BCS class II drug. The main objective of the present investigation was to improve the solubility of itraconazole, by preparation of salt forms itraconazole hydrochloride, mesylate and besylate by using addition reaction with hydrochloric acid, methane sulphonic acid and benzene sulphonic acid. Further inclusion complexes of itraconazole were prepared with Captisol (sulfobutyl ether ${ }_{7} \beta$-cyclodextrin) by using physical mixing, kneading and co-evaporation techniques. The preparations were characterized by using X-ray diffraction, Fourier Transformed Infrared spectroscopy and Nuclear Magnetic Resonance spectroscopy. The solubility of prepared salt was found multifold than the solubility of itraconazole. The dissolution studies exhibited higher percentage drug dissolution from itraconazole complexes than that of the pure drug which can be attributed to the increase in drug solubility provoked by the complexation technique.
\end{abstract}

Keywords Antifungal, Itraconazole, Hydrochloride Salt, Sulfonates Salt, Sulfobutyl Ether 7 B Cyclodextrin, Solubility Enhancement

\section{Introduction}

Poorly water soluble drugs are posing a problem of satisfactory dissolution within the gastro intestinal tract and there by their oral bioavailability. The recent past has witnessed the modern techniques of drug discovery which lead to an increasing number of drug candidates with unfavorable solubility characteristics[1]. Formulation of such compounds for oral delivery has been the most frequent and greatest challenge to scientists in the pharmaceutical industry. Major problem associated with poorly soluble drugs is lack of dissolution there by results in poor and/or variable bioavailability[2]. Kaplan [3] has suggested that the solubility of a drug more than $10 \mathrm{mg} / \mathrm{mL}$ at a $\mathrm{pH}<7$ is expected to have no dissolution as well as bioavailability related problems but, this could be a problem for drugs whose solubility is below $1 \mathrm{mg} / \mathrm{mL}$. Dissolution rate less than $0.1 \mathrm{mg} / \mathrm{cm}^{2} / \mathrm{min}$ were likely to give dissolution rate limited absorption. Solubility of a drug is an intrinsic property and it can only be altered by chemical modification of the molecule by salt formation [4-6] or prodrug formation [7]. Dissolution is an extrinsic property which can be modified by various chemical, physical or crystallographic techniques like complexation, particle size reduction, surface or solid state properties. Different techniques have been reported in the literature for improvement of solubility and drug dissolution rates. These techniques are reduction of the particle size by micronisation or nanonisation to increase the surface area, use of surfactants, Cyclodextrin complexation, pro-drug formation, conversion of crystalline to amorphous forms [8].

Pharmaceutical salts [9] are important in the process of drug development for converting an acidic or basic drug into a salt by a simple neutralization reaction. Using different chemical species to neutralize the parent drug can produce a diverse series of compounds and this process is traditionally being used for modification of the physicochemical, processing, biopharmaceutical or therapeutic properties of drug substances. Each of the individual salts of a particular drug substance can be considered as a unique chemical entity with their own distinctive physicochemical and biopharmaceutical properties [10-12] It has been estimated that approximately half of all of the active pharmaceutical substances (API) that have been developed were ultimately progressed as pharmaceutically acceptable salts and that salt formation is an integral part of the development process $[13,14]$. Sulfonic acid salts particularly alkyl sulfonates such as mesylates and besylates generally results in the formation of high melting point API salts with good solubility and stability[15]. 
Cyclodextrins (CDs) are useful functional excipients that have enjoyed widespread attention and use. A number of cyclodextrin-based products have reached the market based on their ability to change undesirable physicochemical properties of drugs [16,17]. The formation of inclusion complexes provides numerous advantages in pharmaceutical formulation development. $\beta$-CD were reported to increase bioavailability of poorly soluble drugs by increasing the drug solubility [18]. The family of CDs comprises of a series of cyclic oligosaccharides compounds. The three commonly used cyclodextrins are $\alpha$ - cyclodextrins comprised of six glucopyranose units, $\beta$ - cyclodextrins comprised of seven units and $\gamma$-cyclodextrins comprised of eight such units[19]. Sulfobutyl ether $\beta$-Cyclodextrin $\left(\mathrm{SBE}_{7}-\beta-\mathrm{CD}\right)$ [Captisol $\left.{ }^{\circledR}\right]$ [20-22] is a chemically modified $\beta$ - cyclodextrins that is a cyclic hydrophilic oligosaccharide which is negatively charged in aqueous media. The solubility in water for Captisol $(70 \mathrm{~g} / 100 \mathrm{ml}$ at $25 \mathrm{C})$ is significantly higher than the parent $\beta$-cyclodextrin $(1.85 \mathrm{~g} / 100 \mathrm{ml}$ at $25 \mathrm{C})$. It does not exhibit the nephrotoxicity and cytotoxicity which is generally associated with other $\beta$-CDs [23-25]. Some of the investigations also reported that the drug inclusion complex with SBE7- $\beta-C D$ provided a protective effect against drug-induced cytotoxicity [25]. Based on these advantages, Captisol has been selected to study the effect of improving the physiochemical properties of poorly water-soluble drug itraconazole.

Itraconazole (ITR) is a broad-spectrum triazole antifungal agent with poor aqueous solubility [26]. ITR is weakly basic with $\mathrm{pKa}$ of the piperazine ring is 3.7 and highly hydrophobic drug [27]. Because of poor aqueous solubility itraconazole on oral administration results in poor bioavailability and inter individual variations in the plasma drug concentrations. ITR has the characteristic of $\mathrm{pH}$ dependent solubility having highest solubility at acidic side $(4 \mu \mathrm{g} / \mathrm{ml})$ compared to basic $\mathrm{pH}(1 \mu \mathrm{g} / \mathrm{ml})$. However, because of highly liphophilic nature $(\log \mathrm{P}=6.2)$ it can easily penetrate into intestinal membrane. This indicates the poor aqueous solubility is the main reason for lower plasma concentrations. Various techniques [8] have been reported for enhancing the solubility and bioavailability of itraconazole, but the salt formation $[13,14]$ and inclusion complexes [18] showed some promising results. Keeping these in the view the present work was planned with an objective to synthesize Itraconazole hydrochloride, mesylate besylate salt forms from Itraconazole. Further these salt forms were studied for improvement of dissolution by preparing inclusion complexes with Sulfobutyl $_{7}$ Ether $\beta$-Cyclodextrin $\left(\right.$ Captisol $\left.^{\circledR}\right)$ using physical mixing, kneading and co-evaporation techniques. These preparations were characterized by X-ray diffraction, Fourier Transformed Infrared spectroscopy, Nuclear Magnetic Resonance spectroscopy and also evaluated for solubility, drug content and dissolution studies.

\section{Materials and Methods}

Itraconazole was a gift sample obtained from Pharmatech, Hyderabad, and Sulfobutyl ${ }_{7}$ Ether $\beta$-Cyclodextrin $\left(\right.$ Captisol $\left.^{\circledR}\right)$ (average molecular weight 2,163 and degree of substitution 6.5) was obtained from Cydex laboratories. Hydrochloric acid (A.R. grade) Benzene sulfonic acid (A.R. grade) and Methane sulfonic acid (A.R. grade) were purchased from Merck. All other chemicals used in this study were of analytical grade.

\subsection{Preparation of Itraconazole Salts}

Itraconazole hydrochloride (ITRH), Itraconazole mesylate (ITRM) and Itraconazole besylate (ITRB) salts were synthesized from itraconazole (ITR) by acid addition reaction using hydrochloric acid, methane sulfonic acid and benzene sulfonic acid (Figure.1, $2 \& 3$ ). Itraconazole salts were synthesized from a modified method by using acid addition reaction method [28-30]. In case of ITRH preparation, accurately weighed about $1 \mathrm{gm}$ of ITR (1.4 $\mathrm{mmol}$ ) and was dissolved in about $10 \mathrm{ml}$ of dichloromethane in a rotary evaporator flask. To this solution about $400 \mathrm{mg}$ of concentrated hydrochloric acid $(11.42 \mathrm{mmol})$ was added and dissolved. The above suspension was heated at $50{ }^{\circ} \mathrm{C}$ for $1 \mathrm{hr}$ under reflux using rotary evaporator. After one hour $700 \mathrm{mpa}$ vacuum was applied while reaction. The reaction was continued for one hour to form a precipitate of salt. The mixture was allowed to stand overnight at room temperature. The precipitated product was collected, dried at $60^{\circ} \mathrm{C}$ for 1 hour and shifted through \#100 mesh sieve. ITRM and ITRB salts were prepared by following the similar procedure as mentioned above for ITRH salt by taking $1 \mathrm{gm}$ of ITR (1.4 mmol) suspended in about $10 \mathrm{ml}$ of dichloromethane and to this solutions about $400 \mathrm{mg}$ of methane sulfonic acid (4.16 $\mathrm{mmol}$ ) and $600 \mathrm{mg}$ of benzene sulfonic acid $(3.9 \mathrm{mmol})$ were added and dissolved. The final products were stored in an air tight container and then placed in desiccators.

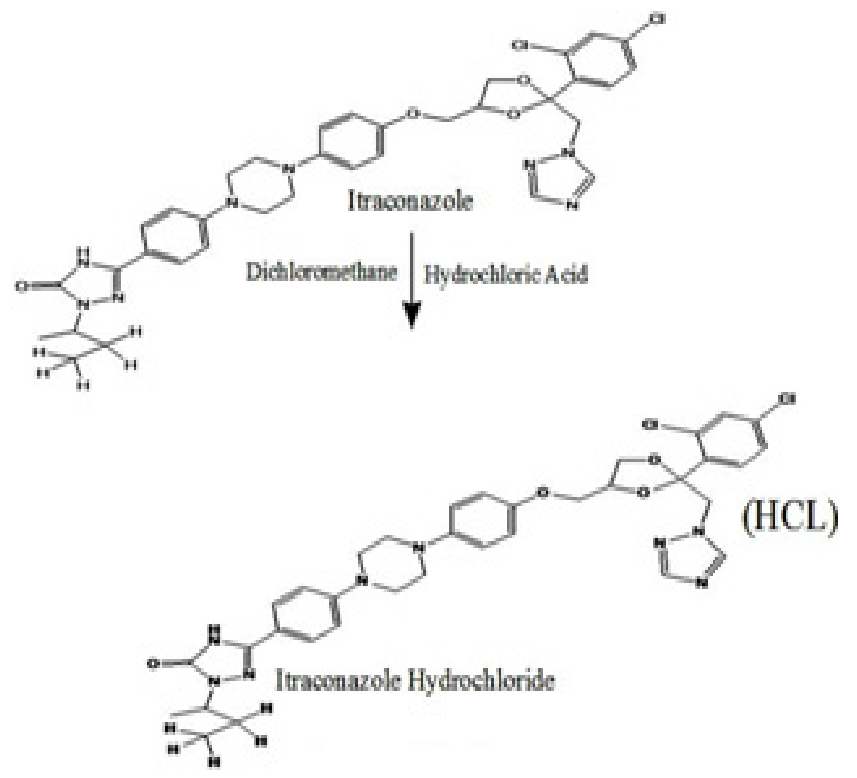

Figure 1. Synthesis of Itraconazole Hydrochloride from Itraconazole 


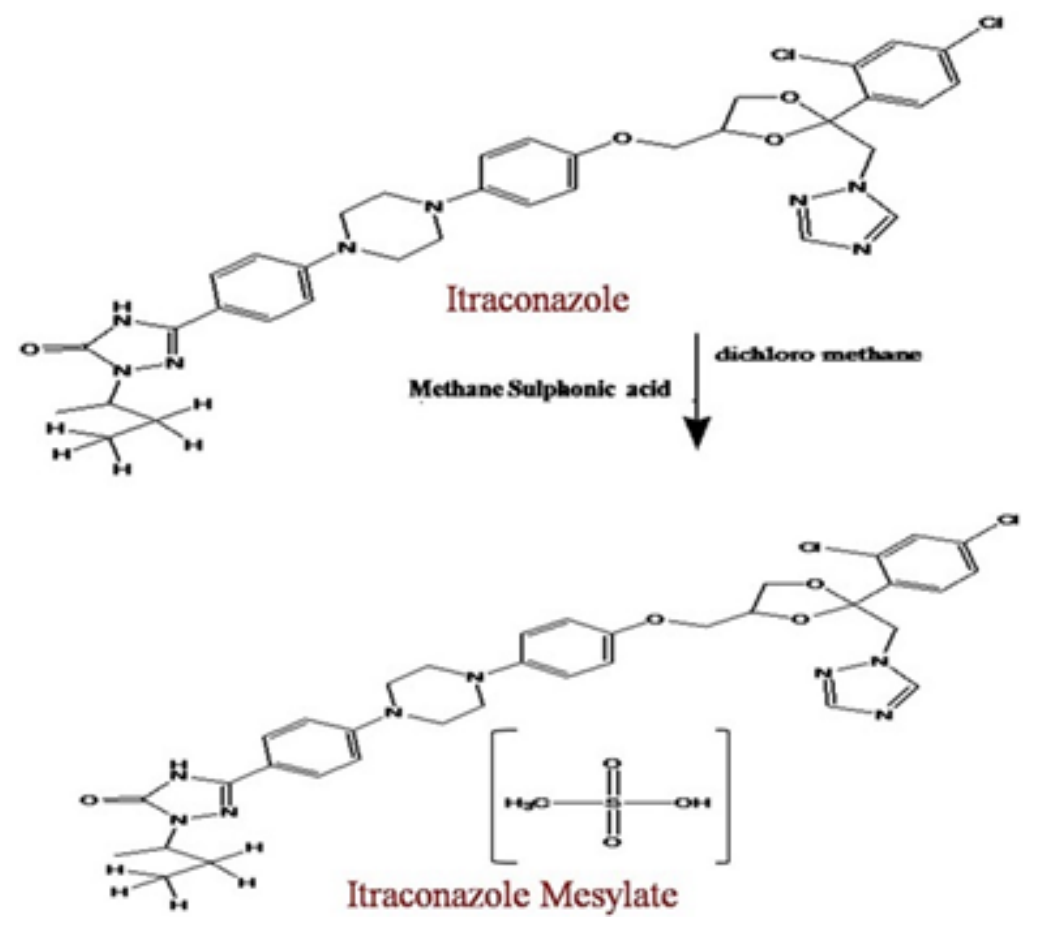

Figure 2. Synthesis of Itraconazole Mesylate from Itraconazole
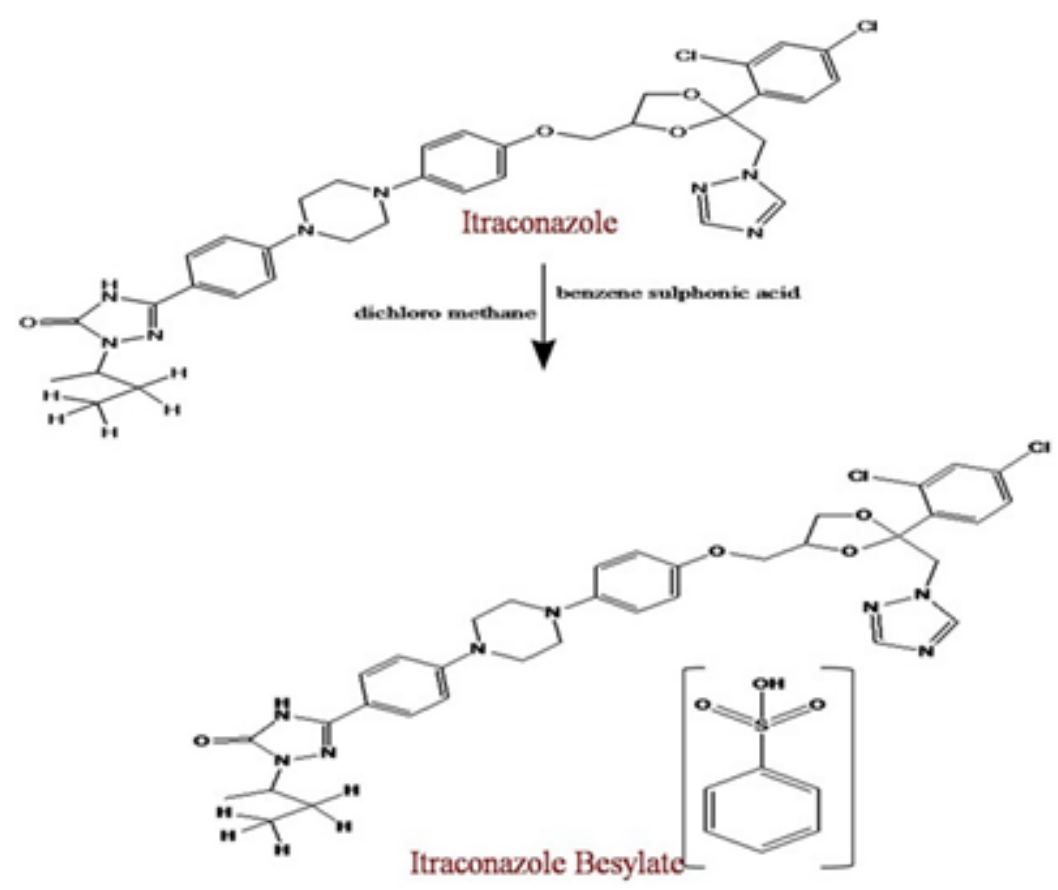

Figure 3. Synthesis of Itraconazole Besylate from Itraconazole

\subsection{Solubility Studies}

Solubility studies for pure ITR, ITRH, ITRM and ITRB were carried in purified water and simulated gastric fluid $(\mathrm{pH}$ 1.2 - $0.1 \mathrm{~N}$ Hydrochloric Acid). In each case excess amount of sample was added to $10 \mathrm{ml}$ of solvent and agitated at $37^{\circ} \mathrm{C}$ in a rotary test tube shaker for $24 \mathrm{hrs}$. After equilibration, the samples were filtered using $0.45 \mu \mathrm{m}$ Millipore filters, suitable diluted and analyzed for the itraconazole content by measuring the absorbance at $258 \mathrm{~nm}$ using Shimadzu
UV-Visible spectrophotometer [31].

\subsection{Phase Solubility Studies}

Phase solubility study [32-36] was carried out to investigate the effect of Captisol on the solubility of ITR, ITRH, ITRM and ITRB using the method reported by Higuchi and Connors. Captisol was added and dissolved in simulated gastric fluid $(\mathrm{pH} \quad 1.2-0.1 \mathrm{~N} \quad \mathrm{HCL})$ to obtain concentrations of $5,10,20,40$ and $80 \mathrm{mM}$. To each of these 
solutions excess amounts of ITR, ITRH, ITRM and ITRB were added separately and shaken using orbit shaker at $25^{\circ} \mathrm{C}$ for $72 \mathrm{hr}$. After equilibrium, the solutions were filtered using $0.45 \mu$ filters and diluted suitably to determine the itraconazole content at $258 \mathrm{~nm}$ using UV-Visible spectrophotometer. The graphs were plotted between solubility of ITR (concentration in $\mathrm{mM}$ ) from pure ITR, ITRH, ITRM and ITRB against the concentration of Captisol (in $\mathrm{mM}$ ). The stability constant for the complex was determined from the graph using the following equation.

$$
K_{s}=\frac{\text { slope }}{s_{0}(1-\text { slope })}
$$

The slope was obtained from the graph and $\mathrm{S}_{0}$ was the equilibrium solubility of ITR, ITRH, ITRM and ITRB in 0.1 $\mathrm{N} \mathrm{HCl}$.

\subsection{Preparation of Inclusion Complexes}

The inclusion complexes of ITR, ITRH, ITRM and ITRB with Captisol (1:2 and 1:3 ratios) were prepared by using physical mixing, kneading and co-evaporation technique [37]. Physical mixture was prepared by simple mixing in a mortar with pestle for $10 \mathrm{~min}$. The powders of ITR, ITRH, ITRM, ITRB and Captisol of required molar ratios are simply mixed in mortar with pestle and then sieved through 100 \#. Kneaded $(\mathrm{KN})$ product was obtained by triturating equimolar quantities of ITR, ITRH, ITRM, ITRB and Captisol of required molar ratios in a mortar with a small volume of solvent blend of water: methanol: dichloromethane at a volume ratio of 2:5:3. During this kneading process few drops of solvent were introduced to maintain a suitable consistency. The resulting mass was dried in an oven at $55^{\circ} \mathrm{C}$ until they get dry and the solid was finally grounded and then sifted through \#100 sieve. In co evaporation technique, aqueous solution of Captisol was added to the solution of ITR, ITRH, ITRM and ITRB in a solvent blend of methanol: dichloromethane at a volume ratio of 2:3.The resultant mixture was stirred for $1 \mathrm{hr}$ and evaporated at a temperature of $55{ }^{\circ} \mathrm{C}$ until dry. The dried mass was pulverized and sifted through \#100 sieve.

\subsection{Fourier Infra Red Spectroscopy (FTIR)}

Fourier transform infrared spectroscopy (FTIR) spectra of ITR, ITRH, ITRM, ITRB, Captisol, ITR-Captisol complexes, ITRH-Captisol complexes, ITRM-Captisol complexes and ITRB-Captisol complexes were recorded using a Fourier Transform Infrared spectrophotometer (Perkin Elmer, Spectrum Two). The samples were scanned from $400-4000$ $\mathrm{cm}^{-1}$ with a resolution of $4 \mathrm{~cm}^{-1}$ by using $\mathrm{KBr}$ pellet technique.

\subsection{X-ray Diffraction Analysis}

Powder X-ray diffraction (XRD) patterns of ITR, ITRH,
ITRM, ITRB, Captisol, ITR-Captisol complexes, ITRH-Captisol, ITRM-Captisol complexes and ITRB-Captisol complexes were recorded on a PAN Analytical X'Pert powder X-ray diffractometer (X-Perto PRO) using Ni-filtered, $\mathrm{Cu} \mathrm{K} \alpha$ radiation, a voltage of $40 \mathrm{kV}$ and $60 \mathrm{~mA}$ current. The scanning rate was $4 \% \mathrm{~min}$ over the diffraction angle range $(2 \theta)$ of $3-50^{\circ}$.

\subsection{NMR Spectroscopy}

The ${ }^{1} \mathrm{H}-\mathrm{NMR}$ spectra of pure ITR, ITRH, ITRM and ITRB were taken in DMSO on a Bruker Ultra shield $400 \mathrm{MHz}$ nuclear magnetic resonance (NMR).Chemical shift values are interpreted for confirmation.

\subsection{Drug Content Estimation}

Accurately weighed $50 \mathrm{mg}$ of the sample and transferred into a $50 \mathrm{ml}$ volumetric flask. Then $25 \mathrm{ml}$ of $50 \%$ methanol:0.1N HCl mixture was added and shaken for 15 minutes to completely dissolve the drug. The volume is made up to $50 \mathrm{ml}$ with $50 \%$ methanol:0.1 $\mathrm{N} \mathrm{HCl}$ mixture. The resulted solution was filtered through $0.45 \mu \mathrm{m}$ filter and suitable diluted and analyzed for the itraconazole content by measuring the absorbance at $258 \mathrm{~nm}$ using Shimadzu UV-Visible spectrophotometer. The drug content of all the inclusion complexes was estimated by following the same method.

\subsection{In vitro Dissolution Studies}

In vitro dissolution studies [38] were carried out in $900 \mathrm{ml}$ of simulated gastric fluid of $\mathrm{pH} 1.2$ using USP Type-II (Paddle) dissolution test apparatus (M/s. Electro Lab India). Sample equivalent to $100 \mathrm{mg}$ of ITR, a speed of $75 \mathrm{rpm}$ and a temperature of $37 \pm 0.5{ }^{\circ} \mathrm{C}$ were used in each test. A $5 \mathrm{ml}$ aliquot was withdrawn at different time intervals, filtered and replaced with $5 \mathrm{ml}$ of fresh dissolution medium. The filtered samples were suitably diluted whenever necessary and assayed for ITR by measuring absorbance at $258 \mathrm{~nm}$. The dissolution studies were carried for the pure ITR and the prepared ITR salts inclusion complexes. Commercial ITR capsules Sporonax ${ }^{\circledR}$ was also evaluated for dissolution for comparison. All the dissolution experiments were conducted in triplicate and the mean values are reported.

\section{Results and Discussion}

\subsection{Nuclear Magnetic Resonance Spectroscopy (NMR)}

NMR spectrum of Itraconazole showed (Figure 4a) the chemical shift values at 0.8(1), 0.9(2), 1.7(3), 1.9(4), 0.8(5), 1.6(6), 3.2(7) and 3.9(8) for methyl, ethyl, N-H, N-H, R-CH2, $\mathrm{C}=\mathrm{C}, \mathrm{C}-\mathrm{Cl}$ and $\mathrm{O}-\mathrm{C} 6 \mathrm{H} 5$ respectively. Itraconazole besylate (Figure 4b) has got chemical shift values at 2.3(1), 3.6(2) for phenyl and $\mathrm{S}=\mathrm{O}$ respectively and Itraconazole mesylate (Figure 4c) has shown at 3.3(1), 4.2(2), 4.3(3), 3.6(4), 1.4(5) 
and 2.3(6) for $-\mathrm{OH},-\mathrm{OH},-\mathrm{OH}, \mathrm{S}=\mathrm{O}, \mathrm{CH} 3-\mathrm{C}=\mathrm{O}$ and phenyl groups respectively. These values indicated the salt conversion of itraconazole into itraconazole mesylate and itraconzole besylate.

\subsection{Solubility Studies}

The solubility of ITR was found to be $1.388 \mu \mathrm{g} / \mathrm{mL}$ in purified water and $7.59 \mu \mathrm{g} / \mathrm{mL}$ in $0.1 \mathrm{~N} \mathrm{HCl}$. The solubility of ITR salts ITRH, ITRM and ITRB in purified water was found to be $23.86 \mu \mathrm{g} / \mathrm{ml}, 165.86 \mu \mathrm{g} / \mathrm{mL}$ and $191.64 \mu \mathrm{g} / \mathrm{mL}$ respectively. The solubility of ITR salts ITRH, ITRM and ITRB in simulated gastric fluid was found to be $93.60 \mu \mathrm{g} / \mathrm{ml}$, $402.6 \mu \mathrm{g} / \mathrm{mL}$ and $508.7 \mu \mathrm{g} / \mathrm{mL}$ respectively. These results clearly indicated that prepared salts have considerable influence on improvement of ITR solubility.

\subsection{Phase Solubility Studies}

The effect of Captisol on the aqueous solubility of ITR,
ITRH, ITRM and ITRB was evaluated using the phase solubility method. The results (Table 1.) showed an increase in the solubility of ITR, ITRH, ITRM and ITRB with increase in Captisol concentration which indicates the effect of complexation. According to Higuchi and Connors, phase solubility study indicated (Figure 5) that the curves can be classified as the AP type (the solubilizer was proportionally more effective at higher concentrations). The positive curvature indicated that the existence of soluble complexes is with an order greater than one. Therefore, the theoretical molar ratio (1:2 and 1:3) were chosen to prepare the solid complexes through different methods. The slope value were lower than one i.e., for ITR, ITRH, ITRM and ITRB was $0.7801,0.035,0.0386$ and 0.0106 respectively.

The apparent stability constant (KS) of ITR: Captisol, ITRH:Captisol, ITRM:Captisol and ITRB: Captisol complex were obtained as $51.322 \mathrm{M}^{-1}, 254.73 \mathrm{M}^{-1}, 213 \mathrm{M}^{-1}$ and 375 $\mathrm{M}^{-1}$ from the initial linear plot of the phase-solubility diagrams.

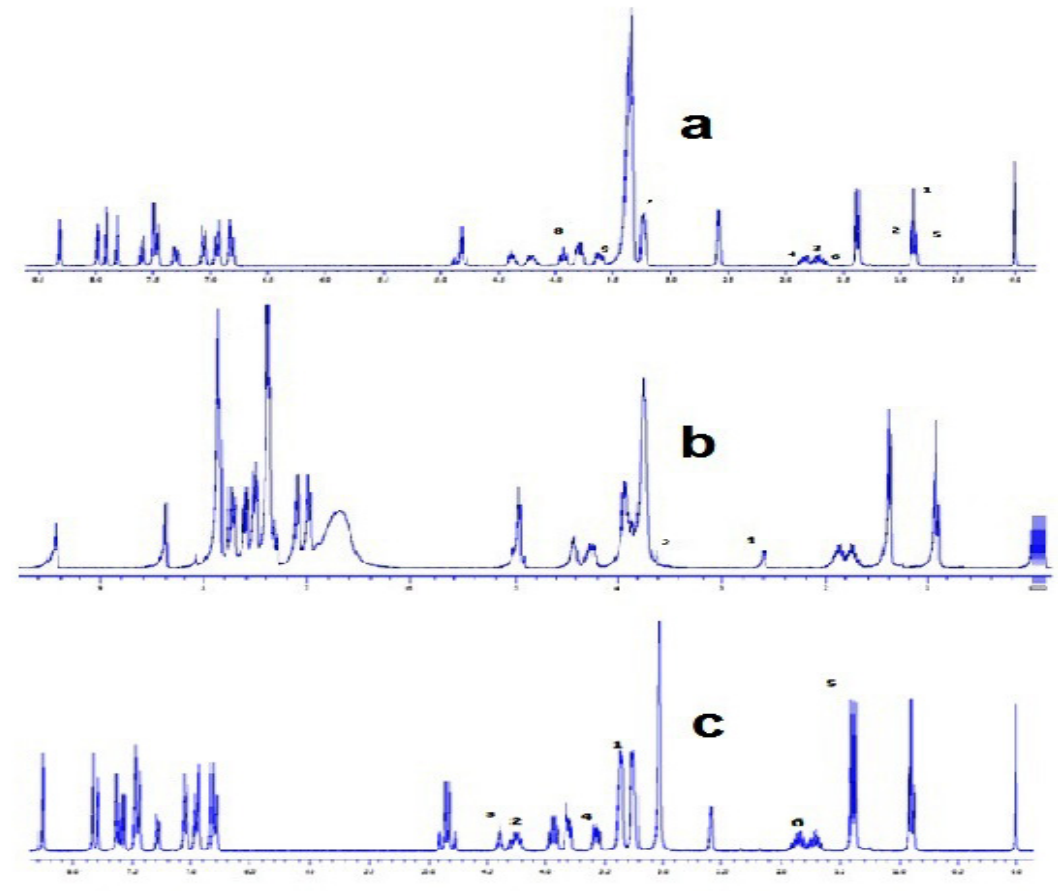

Figure 4. NMR Peaks of (a) Itraconazole (Pure API) (b) Itraconazole Besylate salt (c) Itraconazole Mesylate salt

Table 1. Phase solubility studies

\begin{tabular}{|c|c|c|c|c|c|}
\hline S. No & $\begin{array}{c}\text { Concentration of } \\
\text { Captisol }(\mathrm{mM})\end{array}$ & $\begin{array}{c}\text { Concentration of ITR } \\
(\mathrm{mM})\end{array}$ & $\begin{array}{c}\text { Concentration of } \\
\text { ITRH }(\mathrm{mM})\end{array}$ & $\begin{array}{c}\text { Concentration of } \\
\text { ITRM }(\mathrm{mM})\end{array}$ & $\begin{array}{c}\text { Concentration of } \\
\text { ITRB (mM) }\end{array}$ \\
\hline 1 & 0 & $6.912 \times 10^{-5}$ & 0.00019 & 0.193 & 0.19 \\
\hline 2 & 5 & 0.00312 & 0.359 & 0.386 & 0.616 \\
\hline 3 & 10 & 0.00493 & 0.550 & 0.503 & 1.160 \\
\hline 4 & 20 & 0.00884 & 1.587 & 0.734 & 2.537 \\
\hline 5 & 40 & 0.0222 & 2.589 & 1.658 & 4.253 \\
\hline 6 & 80 & 0.0552 & 34.087 & 3.080 & 7.810 \\
\hline \multicolumn{2}{|c|}{ Stability Constant $\left(\mathrm{k}_{\mathrm{s}}\right)$} & $51.322 \mathrm{M}^{-1}$ & $254.73 \mathrm{M}^{-1}$ & $51.322 \mathrm{M}^{-1}$ & $375 \mathrm{M}^{-1}$ \\
\hline
\end{tabular}




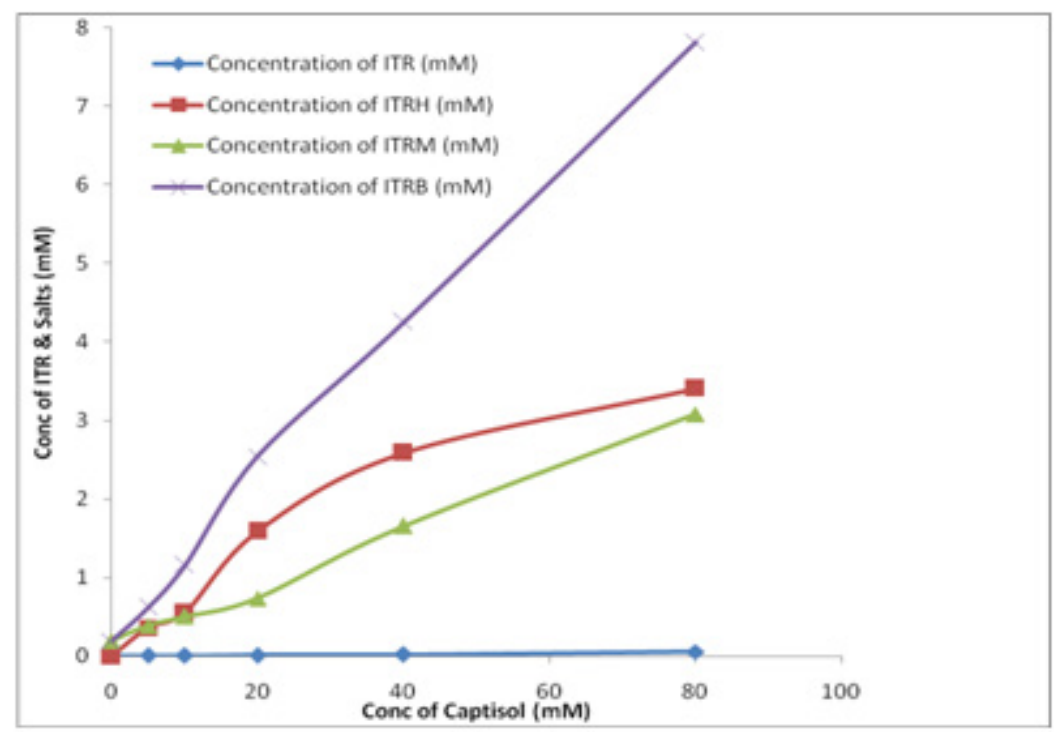

Figure 5. Phase Solubility Graph of ITR and ITR salts with $\mathrm{SBE}_{7} \beta \mathrm{cd}$

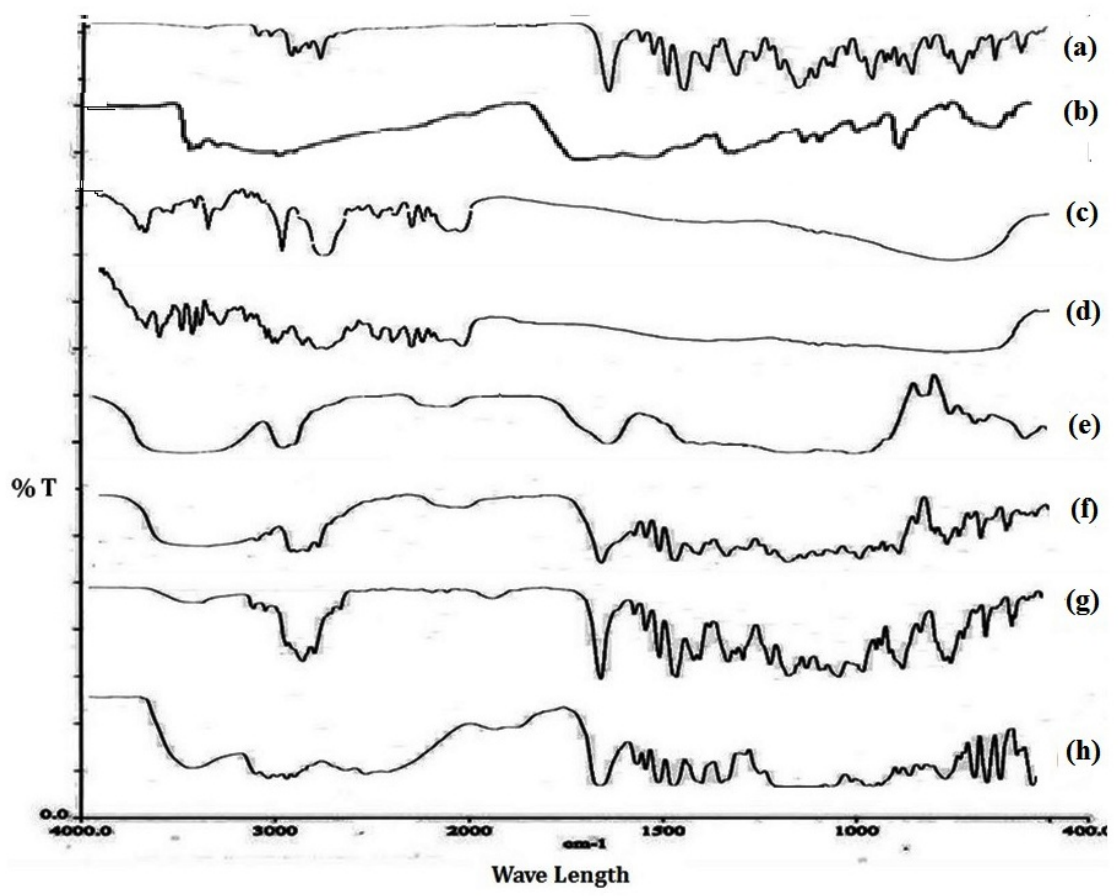

Figure 6. IR spectra of (a) ITR (Pure Drug) (b) ITRH (c) ITRM (d) ITRB (e) Captisol (SBE7 ß-CD) (f) ITRH-C-KN (g) ITRM-C-KN (h) ITRB-C-KN

\subsection{Infra Red Spectroscopy (IR)}

Infra red spectra of pure drug (Figure 6) indicated the presence of characteristic peaks of carboxylate group (O-C-O) in the range of $1550-1660 \mathrm{~cm}-1, \mathrm{C}-\mathrm{N}$ stretch from $1073 \mathrm{~cm}-1$, chlorine group at $700-850 \mathrm{~cm}-1$, benzene moiety from 3100-300 cm-1. The salt forms itraconazole mesylate and itraconazole besylate have got a characteristic peak of $\mathrm{S}=\mathrm{O}$ group in the range of 1345-1365. FTIR studies revealed that Itraconazole HCL showed two typical bands at 3369 and $3283 \mathrm{~cm}-1$ due to N-H primary stretching vibration and a band at $3170 \mathrm{~cm}-1$ due to N-H secondary stretching and characteristics bands at 1623 and $1560 \mathrm{~cm} \mathrm{-1} \mathrm{assigned} \mathrm{to}$
$\mathrm{C}=\mathrm{N}$ stretching. FTIR results suggested that there is no significant chemical interaction between the drug and the Captisol complexed products, which confirms the stability of drug in the powdered form.

\subsection{X-ray Powder Diffraction (XRD)}

The XRD pattern of ITR and ITR complexes samples are shown in Figure 7. The pure drug spectra has shown intense and sharp at 16,20 and $28^{0} 2 \theta$ indicating its crystalline nature. The XRD patterns of salts and the complexed products have been found to have no peaks indicating their amorphous nature and inclusion complex formation with Captisol. 


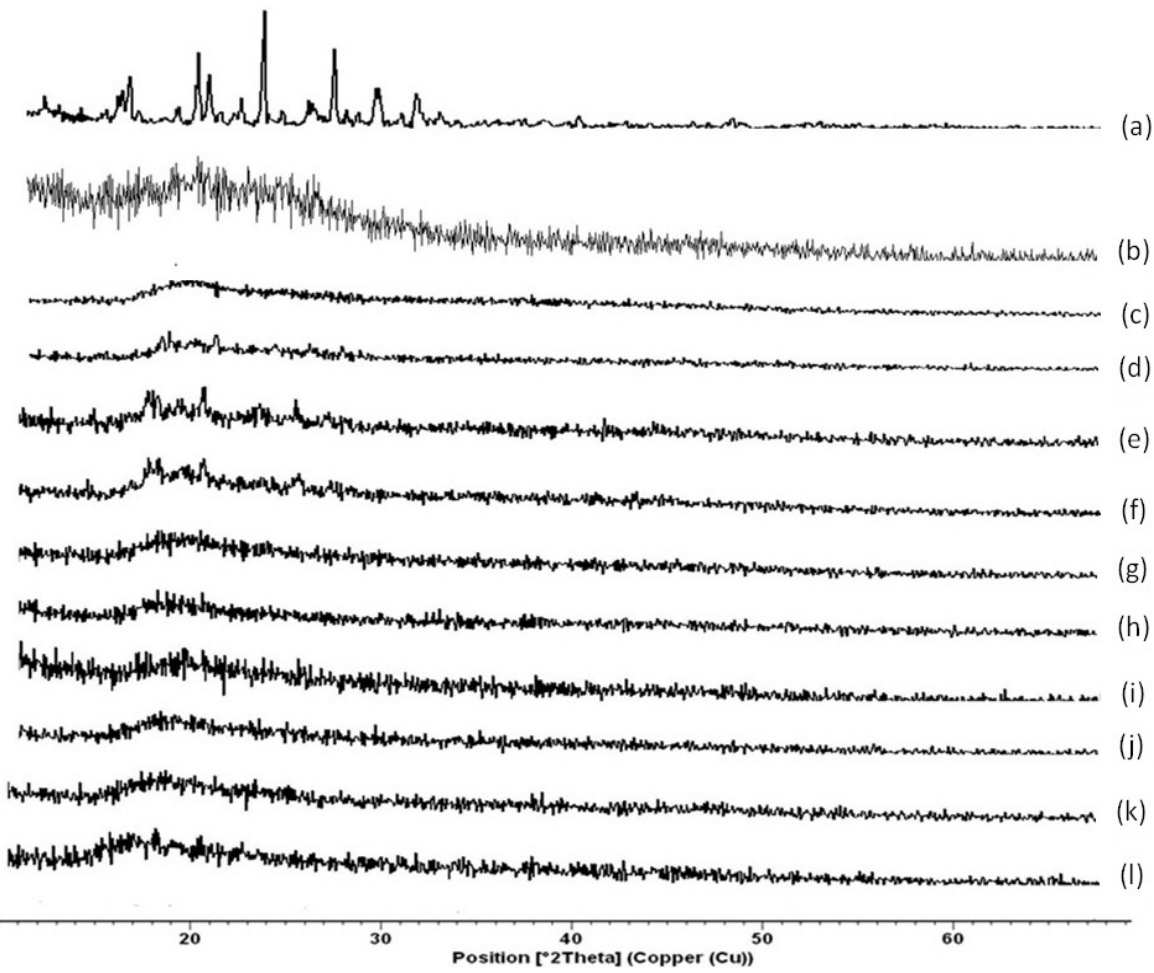

Figure 7. XRD Pattern of (a) ITR (b) ITRM (c) ITRB (d) ITRH (e) ITRH-C-PM (f) ITRH-C-EV (g) ITRH-C-KN (h) ITRM-C-PM (i) ITRM-C-EV (j) ITRM-C-KN(k) ITRB-C-EV (l) ITRB-C-KN

\subsection{Drug Content Estimation}

The percentage drug content of different itraconazole complexes are shown in Table 2 . The drug content was found to be in the range of $75.66 \pm 0.34 \% \mathrm{w} / \mathrm{w}$ to $99.45 \pm 0.18 \% \mathrm{w} / \mathrm{w}$. The low standard deviation values indicated the uniformity of drug content of the prepared complexes.

Table 2. Different itraconazole complexes and their drug content

\begin{tabular}{|c|c|c|c|c|}
\hline \multirow{3}{*}{ Complexes } & \multirow{2}{*}{ Method } & \multirow{2}{*}{ Terminology } & \multicolumn{2}{|c|}{ Drug content $(\% \mathrm{w} / \mathrm{w})$} \\
\cline { 4 - 5 } & & & $1: 2$ & $1: 3$ \\
\hline \multirow{3}{*}{ ITR + C } & $\mathrm{KN}$ & ITR-C-KN & $97.23 \pm 0.12$ & $87.23 \pm 0.17$ \\
\cline { 2 - 5 } & $\mathrm{EV}$ & ITR-C-EV & $93.83 \pm 0.14$ & $81.36 \pm 0.23$ \\
\cline { 2 - 5 } & $\mathrm{PM}$ & ITR-C-PM & $85.89 \pm 0.21$ & $75.66 \pm 0.34$ \\
\hline \multirow{5}{*}{ ITRH + C } & $\mathrm{KN}$ & ITRH-C-KN & $98.45 \pm 0.58$ & $93.86 \pm 0.52$ \\
\cline { 2 - 5 } & $\mathrm{EV}$ & ITRH-C-EV & $94.88 \pm 0.21$ & $91.39 \pm 0.24$ \\
\cline { 2 - 5 } & $\mathrm{PM}$ & ITRH-C-PM & $89.04 \pm 0.41$ & $90.11 \pm 0.45$ \\
\hline & $\mathrm{KN}$ & ITRM-C-KN & $98.64 \pm 0.19$ & $95.64 \pm 0.14$ \\
\cline { 2 - 5 } & $\mathrm{EV}$ & ITRM-C-EV & $94.55 \pm 0.15$ & $94.55 \pm 0.16$ \\
\cline { 2 - 5 } & $\mathrm{PM}$ & ITRM-C-PM & $90.99 \pm 0.12$ & $77.94 \pm 0.33$ \\
\hline \multirow{3}{*}{ ITRB + C } & $\mathrm{KN}$ & ITRB-C-KN & $99.45 \pm 0.18$ & $93.86 \pm 0.25$ \\
\cline { 2 - 5 } & $\mathrm{EV}$ & ITRB-C-EV & $94.88 \pm 0.20$ & $91.39 \pm 0.26$ \\
\cline { 2 - 5 } & $\mathrm{PM}$ & ITRB-C-PM & $89.04 \pm 0.29$ & $80.11 \pm 0.21$ \\
\hline
\end{tabular}

\subsection{In Vitro Dissolution Study of Complexes}

The dissolution profiles of itraconazole from pure drug and different complexes prepared by physical mixture, kneading technique, co-evaporation techniques are shown in Figure $8 \mathrm{a}, 8 \mathrm{~b} \& 8 \mathrm{c}$ respectively.

The pure drug showed the dissolution of $16.89 \%$ in 90 minutes indicating the poor solubility and thereby dissolution. The dissolution of simple physical mixture complexes ITR and Captisol complexes of $1: 2$ and 1:3 weight ratios was found to be $18.86 \%$ and $21.31 \% \mathrm{w} / \mathrm{w}$ respectively, kneading complexes was found to be $32.84 \%$ and $49.87 \% \mathrm{w} / \mathrm{w}$ and co-evaporates was found to be $26.61 \%$ and $42.34 \% \mathrm{w} / \mathrm{w}$ respectively. The data indicated that the only drug complexes with captisol could not able to increase the dissolution to the required level.

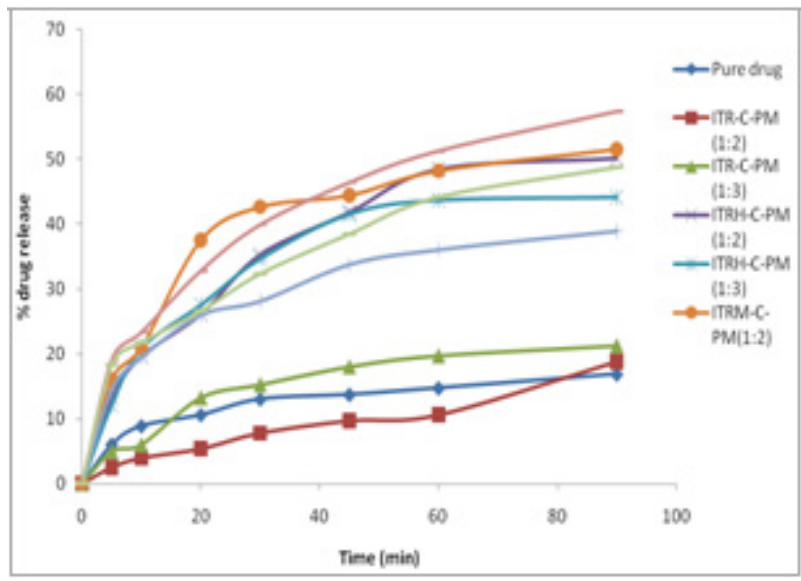

Figure.8a. Percentage drug release of complexes prepared by Physical mixtures method 


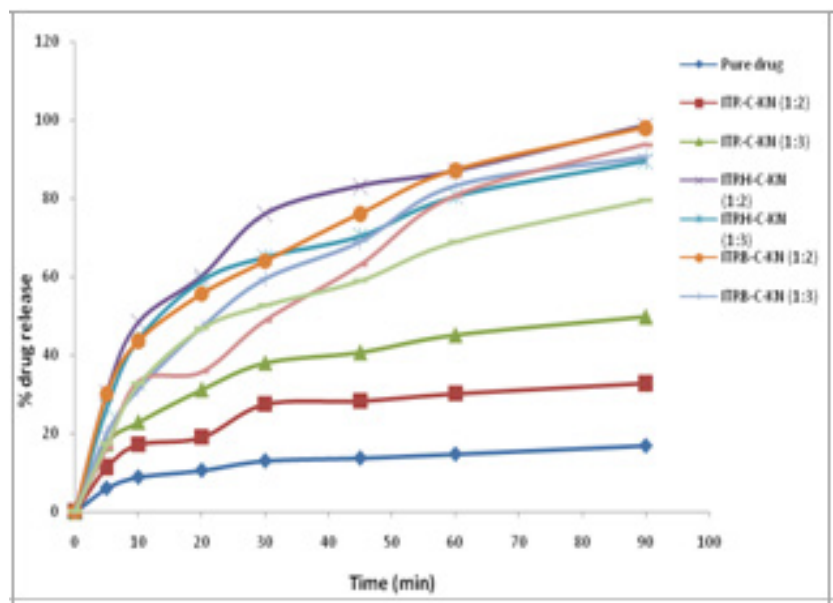

Figure.8b. Percentage drug release of complexes prepared by Kneading mixtures method

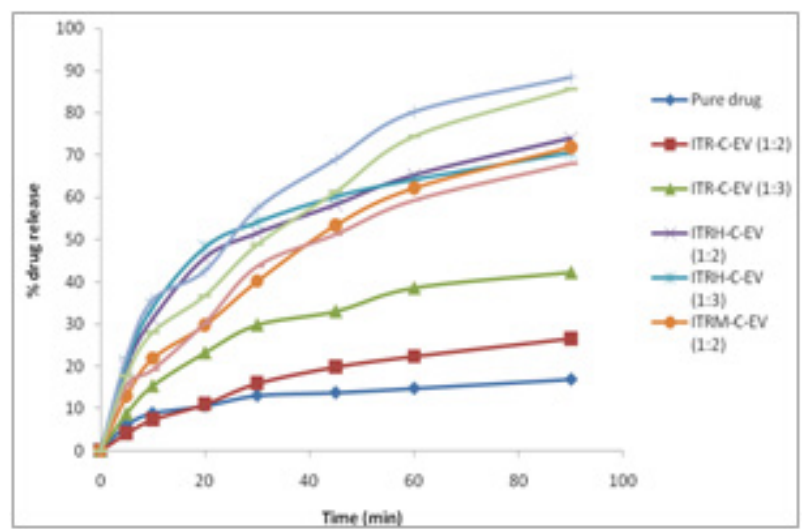

Figure.8c. Percentage drug release of complexes prepared by Co-Evaporation method

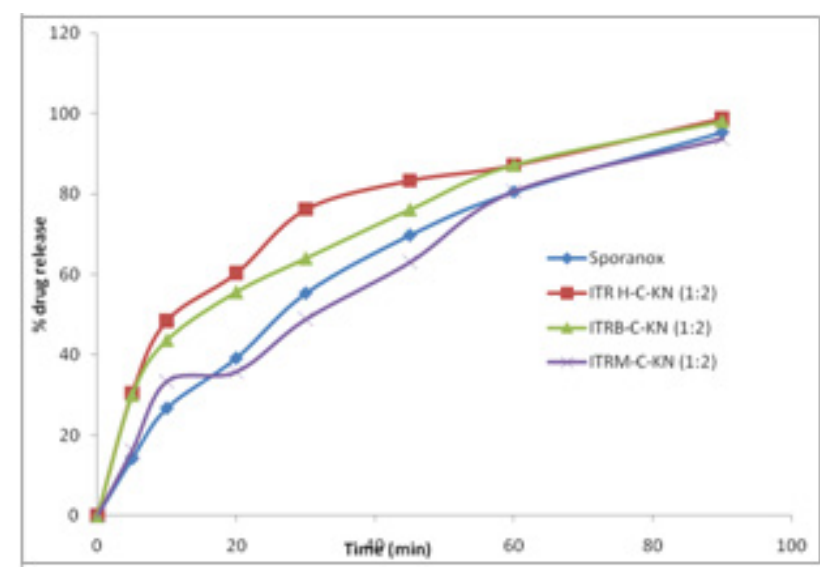

Figure.8d. Percentage drug release of complexes comparison with Sporanox ${ }^{\circledR}$

Further the dissolution of itraconazole salt complexes with captisol at a weight ratio of $1: 2$ and $1: 3$ in physical mixing was found to be $50.12 \%$ and $44.19 \% \mathrm{w} / \mathrm{w}, 51.58 \%$ and $38.90 \% \mathrm{w} / \mathrm{w}$ and $57.36 \%$ and $48.83 \% \mathrm{w} / \mathrm{w}$ respectively for ITRH, ITRM and ITRB. The kneading mixtures showed the dissolution of $98.72 \%$ and $89.72 \% \mathrm{w} / \mathrm{w}, 93.81 \%$ and $79.65 \% \mathrm{w} / \mathrm{w}$ and $98.14 \%$ and $90.73 \% \mathrm{w} / \mathrm{w}$ respectively for ITRH, ITRM and ITRB. The co-evaporation mixtures showed the dissolution of $73.96 \%$ and $70.68 \% \mathrm{w} / \mathrm{w}$,
$71.96 \%$ and $88.43 \% \mathrm{w} / \mathrm{w}$ and $67.92 \%$ and $85.64 \% \mathrm{w} / \mathrm{w}$ respectively for ITRH, ITRM and ITRB. The data clearly indicated that the itraconazole hydrochloride, mesylate and besylate salt complexes with Captisol can significantly increase the dissolution of ITR. For comparison the dissolution of commercial Sporanox capsules dissolution also performed which showed $95.38 \%$ ITR release in 90 minutes (Shown in Figure 8d).

Table 3. Dissolution parameters of Itraconazole

\begin{tabular}{|c|c|c|c|}
\hline Sample & $\begin{array}{c}\text { Dissolution } \\
\text { Efficiency } \\
\text { (DE }\end{array}$ \%) & $\begin{array}{c}\text { Difference } \\
\text { Factor }\left(\boldsymbol{f}_{\mathbf{1}}\right)\end{array}$ & $\begin{array}{c}\text { Similarity } \\
\text { Factor }\left(\boldsymbol{f}_{\mathbf{2}}\right)\end{array}$ \\
\hline ITR & 13.84 & 78 & 17 \\
\hline Sporonax & 69.48 & - & - \\
\hline ITR-C-PM (1:2) & 14.87 & 85 & 16 \\
\hline ITR-C-PM (1:3) & 17.19 & 74 & 18 \\
\hline ITRH-C-PM (1:2) & 40.38 & 38 & 31 \\
\hline ITRH-C-PM (1:3) & 37.69 & 41 & 29 \\
\hline $\begin{array}{c}\text { ITRM-C-PM } \\
(1: 2)\end{array}$ & 43.61 & 33 & 33 \\
\hline $\begin{array}{c}\text { ITRM-C-PM } \\
(1: 3)\end{array}$ & 32.48 & 49 & 25 \\
\hline ITRB-C-PM (1:2) & 45.83 & 32 & 35 \\
\hline ITRB-C-PM (1:3) & 38.85 & 42 & 29 \\
\hline ITR-C-KN (1:2) & 25.75 & 56 & 23 \\
\hline ITR-C-KN (1:3) & 38.04 & 38 & 31 \\
\hline ITRH-C-KN (1:2) & 74.80 & 09 & 62 \\
\hline ITRH-C-KN (1:3) & 72.86 & 17 & 48 \\
\hline $\begin{array}{c}\text { ITRM-C-KN } \\
(1: 2)\end{array}$ & 67.42 & 9 & 64 \\
\hline $\begin{array}{c}\text { ITRM-C-KN } \\
(1: 3)\end{array}$ & 61.86 & 17 & 51 \\
\hline ITRB-C-KN (1:2) & 73.97 & 9 & 65 \\
\hline ITRB-C-KN (1:3) & 70.16 & 10 & 60 \\
\hline ITR-C-EV (1:2) & 19.55 & 72 & 19 \\
\hline ITR-C-EV (1:3) & 33.32 & 50 & 26 \\
\hline ITRH-C-EV(1:2) & 58.88 & 18 & 48 \\
\hline ITRH-C-EV (1:3) & 58.75 & 20 & 46 \\
\hline ITRM-C-EV (1:2) & 52.97 & 23 & 42 \\
\hline ITRM-C-EV (1:3) & 68.68 & 9 & 62 \\
\hline ITRB-C-EV (1:2) & 51.19 & 26 & 40 \\
\hline ITRB-C-EV (1:3) & 63.22 & 11 & 59 \\
\hline
\end{tabular}

The dissolution efficiency $\left(\mathrm{DE}_{90}\right)$ [39] at 90 minutes was calculated and the values are shown in Table 3. Pure itraconazole showed $\mathrm{DE}_{90} \quad 13.84 \%$ and commercial Sporonax capsules showed $69.48 \%$. The salt complexes showed maximum $\mathrm{DE}_{90} 74.80 \%, 67.42 \%$ and $73.97 \%$ for kneading mixtures of ITRH, ITRM and ITRB respectively.

The difference factor $\left(f_{1}\right)$ and similarity factor $\left(f_{2}\right)$ [40] values Shown in Table 3. The results indicated that the salt complexes with captisol prepared by kneading method at weight ratio of 1:2 showed $f_{1}$ values of 9 in all cases of ITRH, ITRM and ITRB and the $f_{2}$ values was 62,65 and 64 respectively. These values indicate the equivalence in dissolution of the prepared complexes with commercial Sporanox capsules. 
Table 4. Dissolution kinetics of Itraconazole

\begin{tabular}{|c|c|c|c|c|c|c|}
\hline Sample & Zero order & First order & Peppas & Higuchi & $\begin{array}{c}\text { First order rate } \\
\text { constant }\left(k_{1}\right)\end{array}$ & n- value \\
\hline ITR & 0.7398 & 0.7633 & 0.9731 & 0.9452 & 0.0017 & 0.3393 \\
\hline Sporonax & 0.9492 & 0.99622 & 0.9899 & 0.9721 & 0.0293 & 0.6897 \\
\hline ITR-C-PM (1:2) & 0.9693 & 0.9680 & 0.9767 & 0.9163 & 0.0021 & 0.6514 \\
\hline ITR-C-PM (1:3) & 0.8105 & 0.8309 & 0.9391 & 0.9626 & 0.0023 & 0.5553 \\
\hline ITRH-C-PM (1:2) & 0.8340 & 0.8890 & 0.9830 & 0.9760 & 0.0060 & 0.485 \\
\hline ITRH-C-PM (1:3) & 0.7490 & 0.8000 & 0.9460 & 0.9410 & 0.0040 & 0.4550 \\
\hline ITRM-C-PM (1:2) & 0.7185 & 0.7873 & 0.9267 & 0.9317 & 0.0069 & 0.4255 \\
\hline ITRM-C-PM (1:3) & 0.7487 & 0.8078 & 0.9857 & 0.9547 & 0.0046 & 0.3487 \\
\hline ITRB-C-PM (1:2) & 0.8215 & 0.9070 & 0.9925 & 0.9827 & 0.0069 & 0.4017 \\
\hline ITRB-C-PM (1:3) & 0.8233 & 0.8958 & 0.9870 & 0.9760 & 0.0046 & 0.3565 \\
\hline ITR-C-KN (1:2) & 0.7296 & 0.7720 & 0.9477 & 0.9306 & 0.0023 & 0.3649 \\
\hline ITR-C-KN (1:3) & 0.7688 & 0.8469 & 0.9857 & 0.9620 & 0.0069 & 0.3712 \\
\hline ITRH-C-KN (1:2) & 0.8930 & 0.9180 & 0.9950 & 0.9970 & 2.3210 & 0.393 \\
\hline ITRH-C-KN (1:2) & 0.7660 & 0.9650 & 0.9520 & 0.9540 & 1.9340 & 0.3970 \\
\hline ITRM-C-KN (1:2) & 0.9311 & 0.9691 & 0.9646 & 0.9762 & 0.0161 & 0.5779 \\
\hline ITRM-C-KN (1:3) & 0.8361 & 0.9654 & 0.9543 & 0.9847 & 0.0161 & 0.4974 \\
\hline ITRB-C-KN (1:2) & 0.9021 & 0.9583 & 0.9922 & 0.9892 & 0.0418 & 0.5546 \\
\hline ITRB-C-KN (1:3) & 0.8592 & 0.9891 & 0.9767 & 0.9890 & 0.0253 & 0.5064 \\
\hline ITR-C-EV (1:2) & 0.9160 & 0.9373 & 0.9875 & 0.9848 & 0.0023 & 0.6526 \\
\hline ITR-C-EV (1:3) & 0.8420 & 0.8908 & 0.9694 & 0.9804 & 0.0046 & 0.5416 \\
\hline ITRH-C-EV (1:2) & 0.810 & 0.937 & 0.973 & 0.974 & 1.658 & 0.4430 \\
\hline ITRH-C-EV (1:3) & 0.743 & 0.873 & 0.946 & 0.943 & 1.547 & 0.4090 \\
\hline ITRM-C-EV (1:2) & 0.9229 & 0.9866 & 0.9928 & 0.9866 & 0.0138 & 0.6005 \\
\hline ITRM-C-EV (1:3) & 0.8660 & 0.9896 & 0.9845 & 0.9855 & 0.0230 & 0.4808 \\
\hline ITRB-C-EV (1:2) & 0.8968 & 0.9693 & 0.9826 & 0.9857 & 0.0115 & 0.5515 \\
\hline ITRB-C-EV (1:3) & 0.9195 & 0.9953 & 0.9945 & 0.9905 & 0.0207 & 0.5490 \\
\hline
\end{tabular}

The kinetics of ITR release from complexes was studied by subjecting the dissolution data to zero order, first order kinetics (as shown in Table 4). The results indicated that the drug release follows first order kinetics. The mechanism of drug release was found to be by diffusion. The correlation values peppas equation indicated the dissolution follows fick's law of diffusion. The study clearly indicated the usefulness of itraconazole hydrochloride, mesylate and besylate salt complexes with sulpho butyl $l_{7}$ ether $\beta \mathrm{CD}$ in improving the solubility and dissolution rate of itraconazole. Among all the complexes the ITRH and ITRB complexes with captisol at a weight ratio of 1:2 prepared by kneading method showed higher dissolution rate.

\section{Conclusions}

The present study showed that itraconazole a poorly soluble drug exhibits very poor in vitro dissolution. The simple drug complexes with captisol also could not able to extend the dissolution rate. The salt form of ITR such as itraconazole hydrochloride, besylate and mesylate salt forms could significantly improve the solubility and dissolution rate of itraconazole. Further the complexation of these salts with captisol has improved the dissolution rate of ITR. Among all the complexes the ITRH and ITRB complexes with captisol at a weight ratio of 1:2 prepared by kneading method showed higher dissolution rate and comparable with commercial Sporanox ${ }^{\circledR}$ capsules.

\section{Acknowledgments}

The Authors are thankful to University Grants Commission, New Delhi for granting financial support to carry the work. The authors also thankful to M/s. Pharmatech, Hyderabad for providing the itraconazole, M/s. TherDose Pharma Private Limited, Hyderabad for providing facilities to conduct these studies. The authors are also thankful to GITAM Institute of Pharmacy, GITAM University for the support. 


\section{REFERENCES}

[1] C. Lipinski. Poor aqueous solubility - An industry wide problem in drug discovery, American Pharmaceutical Review, 5(3), 82-85, 2002.

[2] E. M Merisko-Liversidge, G. G. Liversidge. Drug nanoparticles: formulating poorly water-soluble compounds, Toxicol Pathol, 36(1), 43-48, 2008.

[3] S. A. Kaplan. Biopharmaceutical considerations in drug formulation design and evaluation, Drug Metab. Rev, 1: 15-34, 1972.

[4] G. Tiwari, R. Tiwari. Solid Dispersions: An Overview To Modify Bioavailability Of Poorly Water Soluble Drugs, International Journal of PharmTech Research, 1(4), 1338-1349, 2009.

[5] L. F. Huang, W. Q. Tong. Impact of solid state properties on developability assessment of drug candidates, Adv Drug Deliv Rev, 56, 321-334, 2004.

[6] M. Vasanthavada, A. T. Serajuddin. Lipid-based self-emulsifying solid dispersions, In: Editor David J. Hauss, Oral lipid-based formulations: enhancing the bioavailability of poorly water soluble drugs, 149-183, 2007.

[7] V. J. Stella, K. W. Nti-Addae. Prodrug strategies to overcome poor water solubility. Adavanced Drug delivery reviews, 59, 677-694, 2007.

[8] N. Tiong, A. A. Elkordy. Effects of Liquisolid Formulations on Dissolution of Naproxen, Eur J Pharm Biopharm, 73(3), 373-84, 2009.

[9] A. T. Serajuddin. Salt formation to improve drug solubility. Advanced Drug Delivery Reviews, 59, 603-16, 2007.

[10] S. M. Berge, L. D. Bighley, D. C. Monkhouse. Pharmaceutical salts, J Pharm Sci, 66, 1-19, 1977.

[11] L. D. Bighley, S. M. Berge, D. C. Monkhouse. Salt Forms of Drugs and Absorption, J. Swarbrick, J. C. Boylan. Encyclopaedia of pharmaceutical technology. New York: Marcell-Decker, Vol. 13, pp 453-499, 1995.

[12] P. H. Stahl, G. Wermuth. Monographs on acids and bases.2002. P. H. Stahl, G. Wermuth G, editors. Handbook of Pharmaceutical Salts. Properties, Selection and Use (International Union of Pure and Applied Chemistry). New York: Wiley- VCH. Chapter 12, pp265-327.

[13] S. Balbach, C. Korn. Pharmaceutical evaluation of early development candidates the $100 \mathrm{mg}$ approach, Int J Pharm, 275, 1-12, 2004.

[14] C. R. Gardner, O. Almarsson, H. Chen, S. Morrisette, M. Peterson, Z. Zhang, S. Wang, A. Lemmo, J. Gonzales-Zugasti, J. Monagle, J. Marchionna, S. Ellis, C. McNulty, A. Johnson, D. Levinson, M. J. Cima. Application of a high throughput technology to drug substance and drug product development. Comp Chem Eng, 28, 943-953, 2004.

[15] D. P. Elder, E. D. Delaney, A. Teasdale, S. Eyley, Reif VAND, K. Jacq, F. David. The Utility of Sulfonate Salts in Drug Development, 99(7), 2948-2961, 2010.

[16] A. Biwer, G. Antranikian, E. Heinzle. Enzymatic production of cyclodextrins. Appl Microbiol Biotechnol, 59(6), 609-617, 2002.
[17] K. L. Larsen, L. Duedahl-Olesen, L. H. Pedersen, W. Zimmermann W. Purification and characterization of a cyclodextrin glycosyltransferase from Paenibacillus sp Carbohydrates, 310 (3), 211-219, 1998.

[18] K. A. Connors. Population characteristics of cyclodextrin complex stabilities in aqueous solution, J. Pharm. Sci, 84(3), 843-848, 1995.

[19] I. Maestre, I. Bea, P. Ivanov, C. Jaime. Structural Dynamics of Some Large-Ring Cyclodextrins. A Molecular Dynamics Study: An Analysis of Force Field Performance. Theor. Chem. Acc, 117 (1), 85-97, 2007.

[20] DoeJ.Sulfobutylether-bcyclodextrin.http://www.scribid.com/ doc/77286620/458/ Sulfobutlyether-b-Cyclodextrin.

[21] C. R. Raymond. Hand Book of pharmaceutical Excipients. Pharmaceutical Press and American Pharmacists Association, 6th edition, 714-717, 2009.

[22] James Swarbrick. Encyclopedia of pharmaceutical technology. By Informa Healthcare USA, 3rd Edition, 671-692, 2007.

[23] M. E. Brewster, T. Loftsson. Cyclodextrins as pharmaceutical solubilizers. Adv Drug Deliv Rev, 59, 645-66, 2007.

[24] M. Fukuda, D. Miller, N. Peppas, J. McGinity. Influence of sulfobutyl ether $\beta$-cyclodextrin (Captisol ${ }^{\circledR}$ ) on the dissolution properties of a poorly soluble drug from extrudates prepared by hot-melt extrusion, Int J Pharm, 350,188-96, 2008.

[25] A.M. Tötterman, N. G. M Schipper, D. O. Thompson, J. P. Mannermaa. Intestinal safety of water-soluble $\beta$-cyclodextrins in paediatric oral solutions of spironolactone: effects on human intestinal epithelial Caco-2 cells, J Pharm Pharmacol. 49, 43-48, 1997.

[26] F. C. Odds, M. Oris, P. Van Dorsselaer, F. Van Gerven. Activities of an Intravenous Formulation of Itraconazole in Experimental Disseminated Aspergillus, Candida, and Cryptococcus Infections. Antimicrob. Agents Chemother, 44(11), 3180-3183, 2000.

[27] Jef Peeters, Peter Neeskens, J. P. Tollenaere, Pieter Van Remoortere, M. E. Brewster. Characterization of the interaction of 2-hydroxypropyl- $\beta$-cyclodextrin with itraconazole at $\mathrm{pH} \mathrm{2,} \mathrm{4,} \mathrm{and} \mathrm{7.} \mathrm{J.} \mathrm{of} \mathrm{Pharm.} \mathrm{Sci.,} \mathrm{91(6),}$ 1414-1422, 2002.

[28] N. Kumar, Shishu, V. R. Kapoor. Facile syntheses of novel salts of a triazole antifungal agent with enhanced solubility. J Heterocycl Chem., 50(3), 490-495, 2013.

[29] N. Kumar, Shishu, Gulshan Bansal, Sandeep Kumar, J. Asim Kumar. Ditosylate Salt of Itraconazole and Dissolution Enhancement Using Cyclodextrins, AAPS PharmSciTech., 13(3), 863-874, 2012.

[30] N. Kumar, Shishu, Gulshan Bansal, Sandeep Kumar, J. Asim Kumar. Preparation and cyclodextrin assisted dissolution rate enhancement of itraconazolium dinitrate salt. DrugDevelopment and Industrial Pharmacy, 39(2), 342-51, 2013.

[31] K. P. Shalin, D. P. Ankit, J. B. Dave, C. N. Patel, D. J. Sen. Development and validation of UV spectrophotometric method for estimation of itraconazole bulk drug and pharmaceutical formulation, Int. J. Drug Dev. \& Res., 3(2), 324-328, 2011. 
[32] D. P. Lettre, S. S. Shah, T. Y. Pasha, A. K. Behera, A. Bhandari. Solubility enhancement and physicochemical characterization of inclusion complexes of itraconazole, 4(1), 354-366, 2012.

[33] T. Higuchi, K. A. Connors. Phase solubility techniques. Adv. Anal Chem Instr., 4, 117-212, 1965.

[34] P. María, F. María. Phase solubility studies of terpineol with $\beta$ -cyclodextrins and stability of the freeze- dried inclusion complex, 1, 355-362, 2011.

[35] K. S. Rao, D. B. Udgirkar, D. D. Mule. Enhancement of dissolution rate and bioavailability of Aceclofenac by Complexation with cyclodextrin. Research Journal of Pharmaceutical, Biological and Chemical Sciences, 1(4), 142-151, 2010.
[36] K. J. Waleczek, H. M. C. Marques, B. Hempel, P. C. Schmidt. Phase solubility studies of pure ( 2 ) - a -bisabolol and camomile essential oil with b -cyclodextrin, 55, 247-251, 2003.

[37] K. P. R. Chowdary, S. S. Rao. Investigation of dissolution enhancement of itraconazole by complexation with $\beta$ and hydroxypropyl $\beta$-cyclodextrins, Indian J Pharm Sci., 63, 438441, 2001.

[38] Doe J.Dissolution Methods. FDA-Recommended Dissolution Methods. www.accessdata.fda.gov/scripts/cder/dissolution.

[39] K. A. Khan KA. The concept of dissolution efficiency. J Pharm Pharmacol., 27, 48-9, 1975.

[40] J. W. Moore, H. H. Flanner. Mathematical comparison of dissolution profiles. Pharm. Tech., 20, 64-74, 1996. 